INTEGERS: ELECTRONIC JOURNAL OF COMBINATORIAL NUMBER THEORY 7(2) (2007), \#A07

\title{
SOME PROBLEMS IN VIEW OF RECENT DEVELOPMENTS OF THE ERDÖS GINZBURG ZIV THEOREM
}

\author{
Arie Bialostocki \\ Department of Mathematics, University of Idaho,Moscow, ID 83844 US \\ arieb@uidaho.edu
}

Received: 1/16/06, Revised: 7/17/06, Accepted: 8/6/06

\begin{abstract}
Two conjectures concerning the Erdős Ginzburg Ziv theorem were recently confirmed. Reiher and di Fiore proved independently the two dimension analogue of the EGZ theorem, as conjectured by Kemnitz, and Grynkiewicz proved the weighed generalization of the EGZ theorem as conjectured by Caro. These developments trigger some further problems. First, we will present computer experiments that at least for small numbers reveal very simple phenomena of zero sum theorems that seem to be difficult to prove. Next, we will examine the notion of generalization of Ramsey type theorems in the sense of a given zero sum theorem in view of the new developments.
\end{abstract}

\section{Introduction}

We begin by recalling the extended version of the Erdös Ginzburg Ziv theorem known today as the EGZ theorem.

\section{Theorem 1}

(a) Suppose $G=(G,+, 0)$ is an abelian group of order $n$. Let I be a set of indices and let $\left\{a_{i}\right\}_{i \in I}$ be an indexed set of elements from $G$. If $|I|=2 n-1$, then there exists a subset $J \subset I$, with $|J|=n$, such that $\sum_{j \in J} a_{j}=0$.

(b) Furthermore, if $|I|=2 n-2$ and there does not exist a subset $J \subset I$, with $|J|=n$, such that $\sum_{j \in J} a_{j}=0$, then (b)(i) and (b)(ii) hold:

(i) The group $G$ is cyclic. 
(ii) Half of the $a_{i}$ 's are equal to $x \in G$, the other half of the $a_{i}$ 's are equal to $y \in G$, where $x-y$ is a generator of $G$.

Part (a) was originally proved in [20] for cyclic groups only, but it is not difficult to deduce the proof for any abelian group. Several other proofs appear in [2] and another one in [35]. Part (b) is known as a solution to an inverse problem. The proofs of parts (b)(i) and (b)(ii) can be deduced from [7] and [16], respectively. See also [40].

The author is interested mainly in generalizations of extremal theorems from combinatorics in the sense of a given zero sum theorem. But what is a zero sum theorem? To define rigorously what is a zero sum theorem, is quite a challenging task, and such an attempt will require the introduction of some intricate notation. But more disturbing will be that after our efforts, some new zero sum theorems in the philosophical sense will be discovered that are not covered by our definition. Thus, we prefer to accept the intuitive notion of a zero sum theorem. Similarly to Ramsey Theory being philosophically defined as "total disorder is impossible" we say that "avoiding zero configurations is impossible." First let's look at some zero sum theorems. The following one is known as the cave's man theorem, [21].

Theorem 2 Suppose $G=(G,+, 0)$ is an abelian group. If $S$ is a sequence of elements from $G$ of length $|G|$, then $S$ contains a subsequence all of whose elements add up to zero.

Though, Theorem 2 falls into the category of a zero sum theorem, however in the foregoing we will be interested in theorems that resemble more the EGZ theorem. Here are three important characteristics of the EGZ theorem.

\section{Observation 3}

(a) The number of elements involved in the "zero sum configuration" is predetermined, say $n$. In the EGZ theorem the number $n$ is the order of the group.

(b) If the same element from $G$ appears in the sequence $n$ times, then the conclusion of the theorem follows trivially.

(c) If the number of distinct elements in the sequence is bounded by some number, then the conclusion of the theorem follows easily. In the EGZ theorem this number is two, and in this case the EGZ theorem follows from the pigeon-hole principle.

We will demonstrate another zero sum theorem, Theorem 5, which has the above characteristics. But first we need to introduce some definitions. 
Definition 4 Let $n$ and $r$ be two positive integers. Define Union $\left(\mathbb{Z}_{n}^{r}\right)$ to be the union of $r$ disjoint copies of $\mathbb{Z}_{n}$ where addition can be performed within each copy only.

$$
\operatorname{Union}\left(\mathbb{Z}_{n}^{r}\right)=\underbrace{\mathbb{Z}_{n} \dot{\cup} \mathbb{Z}_{n} \dot{\cup} \ldots \dot{\cup} \mathbb{Z}_{n}}_{r \text { times }} \text { and } \operatorname{Union}^{+1}\left(\mathbb{Z}_{n}^{r}\right)=\underbrace{\mathbb{Z}_{n} \dot{\cup} \mathbb{Z}_{n} \dot{\cup} \ldots \dot{\cup} \mathbb{Z}_{n}}_{r \text { times }} \cup\{1\},
$$

where $1 \in \mathbb{Z}_{n}$.

\section{Theorem 5}

(a) If $S$ is a sequence with elements from Union $\left(\mathbb{Z}_{n}^{r}\right)$, of length $2 r(n-1)+1$, then $S$ contains a subsequence of length $n$, all whose elements add up to zero.

(b) If $S$ is a sequence with elements from $\operatorname{Union}^{+1}\left(\mathbb{Z}_{n}^{r}\right)$, of length $(2 r+1)(n-1)+1$, then $S$ contains a subsequence of length $n$, all whose elements add up to zero.

The proof of Theorem 5 follows easily from the pigeon-hole principle and the EGZ theorem. Furthermore, if in part (a), $r=1$, then we get the EGZ theorem. It is worthwhile to mention that Theorem 5 generalizes the following form of the pigeon principle: "If $2 r(n-1)+1$ $((2 r+1)(n-1)+1)$ pigeons are distributed into $2 r$ in part (a) $(2 r+1$ in part (b)) holes, then there are at least $n$ pigeons in one of the holes."

In Section 2 we focus on three generalizations of the EGZ theorem. Two of them have been recently proved and the third one is still an open problem, but some recent progress has been made. In Section 3 we suggest a new line of zero sum theorems and make several conjectures. In Section 4 we introduce the notion of generalization in the sense of the EGZ theorem, but with some modification the reader can define the notion of generalization in the sense of other zero sum theorems.

\section{On Three Generalizations of the EGZ Theorem}

The general background reference to these kinds of problems in additive number theory is [39]. Many generalizations of the EGZ theorem have been proved and many others are still open problems. For example, see [11], [15], [24], [28], [44] and [46]. There have been many other recent developments of the EGZ theorem, for example see [1], [9], [25], [32], [33], [47] and [48]. In this section we will focus only on three generalizations, not mentioned above. We begin with the multiplicity conjecture conjectured in [5].

Conjecture 6 Suppose $s$ and $n$ are two integers, where $n \geq 2$. If $a_{1}, a_{2}, \ldots, a_{s}$ is a sequence of elements from $\mathbb{Z}_{n}$, then there are at least $\left(\begin{array}{c}\left\lfloor\frac{s}{2}\right. \\ n\end{array}\right)+\left(\begin{array}{c}\left\lceil\frac{s}{2}\right\rceil \\ n\end{array}\right)$ subsets $I \subset\{1, \ldots, s\}$, such that $|I|=n$ and $\sum_{j \in I} a_{j}=0$. 
If in Conjecture 6, we substitute $s=2 n-1$, then the EGZ theorem follows. In [37] the conjecture has been proved if $n \in\left\{p^{\alpha}, p^{\alpha} q\right\}$, where $p$ and $q$ are primes. Furthermore, in [23] it has been proved asymptotically for a fixed $n$ and $s \rightarrow \infty$. And recently it was proved in [30] for $s \leq 6 \frac{1}{3} n$.

The second generalization of the EGZ theorem Theorem 7, was proved by Grykiewicz in [31]. The theorem was conjectured by Caro [17].

Theorem 7 (CG) Let $w_{1}, w_{2}, \ldots, w_{n} \in \mathbb{Z}_{n}$ and suppose $\sum_{i=1}^{n} w_{i}=0$. If $a_{1}, a_{2}, \ldots, a_{2 n-1}$ is a sequence of elements from $\mathbb{Z}_{n}$, then there exists a subset $J \subset\{1,2, \ldots, 2 n-1\}$, with $|J|=n$, such that with some rearrangement of the corresponding subsequence we get: $\sum_{k \in I, i=1}^{n} a_{k} w_{i}=$ 0 .

If in Theorem 7 we have $w_{i}=1$ for every $i$, then the EGZ theorem follows.

The third generalization of the EGZ theorem was proved independently by Reiher [41] and di Fiore as reported in [45]. The theorem was conjectured by Kemnitz [36].

Theorem 8 (KFR) Every sequence of elements from $\mathbb{Z}_{n} \oplus \mathbb{Z}_{n}$ of length $4 n-3$ contains a subsequence of length $n$, such that all of its elements add up to 0.

It is worthwhile to comment that Theorem 8 can be viewed as a two dimensional generalization of the EGZ theorem. However, the innocent extrapolation for higher dimensions does not work, [19]. For a unified approach to the EGZ theorem and the KFR theorem see [18].

We conclude this section with the multiplicity weighted version of Theorem 8 .

Conjecture 9 Suppose $s$ and $n$ are two integers, where $n \geq 2$, and let $s=4 k+r$, where $r \in$ $\{0,1,2,3\}$. Furthermore, suppose $w_{1}, w_{2}, \ldots, w_{n} \in \mathbb{Z}_{n}$ satisfy $\sum_{i=1}^{n} w_{i}=0$. If $a_{1}, a_{2}, \ldots, a_{s}$ is a sequence of elements from $\mathbb{Z}_{n} \oplus \mathbb{Z}_{n}$, then there are at least $(4-r)\left(\begin{array}{l}k \\ n\end{array}\right)+r\left(\begin{array}{c}k+1 \\ n\end{array}\right)$ subsequences $I \subset\{1,2, \ldots, s\}$, such that $|I|=n$, and $\sum_{k \in I, i=1}^{n} a_{k} w_{i}=0$.

\section{Conjectures About Other Zero Sum Theorems}

The novelty of Theorem 7 is that we look at $\mathbb{Z}_{n}$ as a ring or a module and not just a group and it suggests many other theorems along the same line. We make the following conjectures. 
Conjecture 10 Let $\mathbb{Z}_{n}$ be the ring of all residues modulo $n$. Define $f(n)$ to be the smallest positive integer such that every sequence of elements from $\mathbb{Z}_{n}$ of length $f(n)$ contains a subsequence $a_{1}, \ldots, a_{n}$ and a permutation $\sigma$ such that $\sum_{i=1}^{n} a_{i}=0$ and $\sum_{i=1}^{n} a_{i} a_{\sigma(i)}=0$. Then

$$
f(n)=\left\{\begin{array}{ll}
2 n+1 & \text { if } n=3 \quad(\bmod 4) \\
2 n-1 & \text { otherwise }
\end{array} .\right.
$$

If $n=3(\bmod 4)$, then the extremal sequence is $0^{n-1} 1^{2} 2^{n-1}$. If $n \neq 3(\bmod 4)$, then the extremal sequence is $0^{n-1} 1^{n-1}$.

The conjecture was confirmed for $n \leq 13$.

Conjecture 11 Let $\mathbb{Z}_{n}$ be the ring of all residues modulo $n$, and let $u_{i}$ and $w_{i}$, for $i=$ $1,2, \ldots, n$ be elements of $\mathbb{Z}_{n}$, satisfying $\sum_{i=1}^{n} u_{i}=0$ and $\sum_{i=1}^{n} w_{i}=0$. Define $f(n)$ to be the smallest positive integer such that every sequence of elements from $\mathbb{Z}_{n}$ of length $f(n)$ contains a subsequence $a_{1}, a_{2}, \ldots, a_{n}$ and two permutations $\sigma$ and $\theta$ such that $\sum_{i=1}^{n} u_{i} a_{\sigma(i)}=0$ and $\sum_{i=1}^{n} w_{i} a_{\theta(i)}=0$. Then

$$
f(n)=\left\{\begin{array}{ll}
2 n+1 & \text { if } n \text { is odd } \\
2 n-1 & \text { if } n \text { is even }
\end{array} .\right.
$$

If $n$ is even, then the extremal sequence is $0^{n-1} 1^{n-1}$. If $n$ is odd, then the extremal sequence is $0^{n-1} 1^{n-1} 2^{1}(n-1)^{1}$.

The conjecture was confirmed for $n \leq 7$.

Conjecture 12 Let $\mathbb{Z}_{n}$ be the ring of all residues modulo $n$. Define $f(n)$ to be the smallest positive integer such that every sequence of elements from $\mathbb{Z}_{n}$ of length $f(n)$ contains two disjoint subsequences $a_{1}, a_{2}, \ldots, a_{n}$ and $b_{1}, b_{2}, \ldots, b_{n}$ such that $\sum_{i=1}^{n} a_{i}=\sum_{i=1}^{n} b_{i}=0$ and $\sum_{i=1}^{n} a_{i} b_{\sigma(i)}=0$, for some permutation $\sigma$. Then $f(n)=3 n-1$. The extremal sequence is given by $0^{2 n-1} 1^{n-1}$ and it is unique.

The conjecture was confirmed for $n \leq 12$.

Conjecture 13 Let $\mathbb{Z}_{n}$ be the ring of all residues modulo $n$. Define $f(n)$ to be the smallest positive integer such that every sequence of elements from $\mathbb{Z}_{n}$ of length $f(n)$ contains three pairwise disjoint subsequences $a_{1}, a_{2}, \ldots, a_{n}, b_{1}, b_{2}, \ldots, b_{n}, c_{1}, c_{2}, \ldots, c_{n}$ and permutations $\alpha, \beta, \gamma, \sigma, \theta$ such that:

$$
\sum_{i=1}^{n} a_{i}=\sum_{i=1}^{n} b_{i}=\sum_{i=1}^{n} c_{i}=\sum_{i=1}^{n} a_{i} b_{\alpha(i)}=\sum_{i=1}^{n} a_{i} c_{\beta(i)}=\sum_{i=1}^{n} b_{i} c_{\gamma(i)}=\sum_{i=1}^{n} a_{i} b_{\sigma(i)} c_{\theta(i)}=0 .
$$

Then $f(n)=4 n-1$. The extremal sequence is given by $0^{3 n-1} 1^{n-1}$, or by $0^{2 n-1} 1^{2 n-1}$ and it is unique. 
The conjecture was confirmed for $n \leq 9$.

Conjecture 14 Let $\mathbb{Z}_{n}$ be the ring of all residues modulo $n$. Suppose $a_{1}, a_{2}, \ldots, a_{n}$ and $b_{1}, b_{2}, \ldots, b_{n}$ for $i=1,2, \ldots, n$ are sequences of elements from $\mathbb{Z}_{n}$, satisfying $\sum_{i=1}^{n} a_{i}=$ $\sum_{i=1}^{n} b_{i}=0$. If $n$ is even, then there exists a permutation $\alpha$ such that $\sum_{i=1}^{n} a_{i} b_{\alpha(i)}=0$.

The conjecture was confirmed for $n \leq 8$.

It is worthwhile to comment that if Conjecture 14 holds true, then for $n$ even it puts Theorem 7 in a new light. We conclude with Conjecture 15 that may seem obscure at first glance, but its significance will be seen in the next section.

Conjecture 15 Let $\mathbb{Z}_{n}$ denote the additive cyclic group of the set of residues $\{0,1, \ldots, n-1\}$, with the metric $d(x, y)=\min \{|x-y|,|n-(x-y)|\}$. Denote by $f(n)$ the smallest integer such that such that every sequence of elements from $\mathbb{Z}_{n}$ of length $f(n)$ contains an $n$-element subsequence that can be rearranged as $a_{1}, a_{2}, \ldots, a_{n}\left(a_{n+1}=a_{1}\right)$ to satisfy $\sum_{i=1}^{n} d\left(a_{i+1}, a_{i}\right)=$ 0. Then

$$
f(n)= \begin{cases}3 n-2 & \text { if } n \text { is odd } \\ \frac{3}{2} n-1 & \text { if } n \text { is even }\end{cases}
$$

The lower bound is given by $0^{n-1} 1^{n-1} 2^{n-1}$ and $0^{n-1} 1^{n / 2-1}$ (not unique) for $n$ odd and even respectively. The conjecture was confirmed for $n \leq 9$ and $n \leq 12$ for $n$ odd and even respectively.

\section{Generalization in the Sense of a Zero Sum Theorem}

In this section, we first introduce the definitions of Ramsey numbers for graphs and generalized Rado numbers, as well as their zero sum counterparts. These definitions enable us to define generalization in the sence of Theorem 1.5. Similar definitions can be applied to other extremal numbers, like Turan numbers for example, their zero sum counterparts and generalizations in the sence of agiven zero sum theorem. The general reference for Ramsey theory is [26]. However we have a significant interest along the lines of [38]. Ramsey theory on the integers started by Schur and was developed by Rado in the thirties. It mainly deals with monochromatic solutions to general systems of linear equations. The interest of integrating linear inequalities into Ramsey theory on the integers is pretty new, [34] and [42]. After introducing the definitions we provide some references to generalizations in the sence of the Theorem 1.5, (mainly its particular case, the EGZ theorem). We will conclude the section with the notion of multiplicity Ramsey and Rado numbers, their zero sum counter parts, and generalizations in the sense of Conjecture 2.1. 
Definition 16 (Ramsey numbers for graphs) Let $\mathcal{G}$ be a family of graphs each having $n$ edges and let $t$ be a positive integer. Denote by $R(\mathcal{G}, t)\left(R_{E G Z}\left(\mathcal{G}, U n i o n\left(\mathbb{Z}_{n}^{t / 2}\right)\right)\right.$, if $t$ is even and $R_{E G Z}\left(\mathcal{G}\right.$, Union $\left.^{+1}\left(\mathbb{Z}_{n}^{(t-1) / 2}\right)\right)$ if $t$ is odd) the minimum number $s$ such that if the edges of the complete graph on $s$ vertices, $K_{s}$, are colored by $t$ colors, (are colored by Union $\left(\mathbb{Z}_{n}^{t / 2}\right.$ ) if $t$ is even and by Union ${ }^{+1}\left(\mathbb{Z}_{n}^{(t-1) / 2}\right)$ if $t$ is odd), then there exists a copy in $K_{s}$ that is isomorphic to an element of $\mathcal{G}$, such that it is monochromatic (the colors of its edges add up to zero).

Definition 17 (Rado numbers) Let $x_{1}, x_{2}, \ldots, x_{n k}$ be $n k$ variables partitioned into $k$ sets $S_{i}$, where $i=1,2, \ldots, k$, and suppose that $\left|S_{i}\right|=n$. Furthermore, let $\mathcal{L}$ be a system that consists of equations or inequalities in the variables $x_{1}, x_{2}, \ldots, x_{n k}$. For a positive integer $t$ denote by $R(\mathcal{L}, t) \quad\left(R_{E G Z}\left(\mathcal{L}, \operatorname{Union}\left(\mathbb{Z}_{n}^{t / 2}\right)\right)\right.$, if $t$ is even and $R_{E G Z}\left(\mathcal{L}, \operatorname{Union}^{+1}\left(\mathbb{Z}_{n}^{(t-1) / 2}\right)\right)$ if $t$ is odd) the minimum number s such that if the integers $1,2, \ldots, s$ are colored by $t$ colors, (are colored by Union $\left(\mathbb{Z}_{n}^{t / 2}\right)$ if $t$ is even and by Union ${ }^{+1}\left(\mathbb{Z}_{n}^{(t-1) / 2}\right)$ if $t$ is odd), then there exist integers $x_{1}, x_{2}, \ldots, x_{n k}$, which satisfy $\mathcal{L}$ and each $S_{i}$ is monochromatic, but not necessarily the same color (the colors of the variables in each $S_{i}$ add up to zero).

In order to convey the ideas clearly, we have limited the scope of our definitions. For instance our definition does neither include the off diagonal case nor a disjunctive system of equations and inequalities.

Definition 18 (Generalization in the sense of Theorem 5) We say that a theorem concerning Ramsey numbers, (Rado numbers) admits a generalization in the sense of Theorem 5 , if $R(\mathcal{G}, t)=R_{E G Z}\left(\mathcal{G}, \operatorname{Union}\left(\mathbb{Z}_{n}^{t / 2}\right)\right)$, if $t$ is even and $R(\mathcal{G}, t)=R_{E G Z}\left(\mathcal{G}\right.$, Union $\left.^{+1}\left(\mathbb{Z}_{n}^{(t-1) / 2}\right)\right)$ if $t$ is odd, $\left(R(\mathcal{L}, t)=R_{E G Z}\left(\mathcal{L}, \operatorname{Union}\left(\mathbb{Z}_{n}^{t / 2}\right)\right)\right.$, if $t$ is even and $R(\mathcal{L}, t)=R_{E G Z}\left(\mathcal{L}\right.$, Union $\left.^{+1}\left(\mathbb{Z}_{n}^{(t-1) / 2}\right)\right)$ if $t$ is odd. $)$

Generalizations in the sense of Theorem 1,( Theorem 1 is included in Theorem 5), that relate to graph theory appear in [4],[7],[8][22] and [43]. The two most handled systems of linear equeation are those which follow from the Schur equation $x+y=z$ and the arithmetic progression, known as the Van der Waerden problem. There have been various variations and modifications of the Schur equation and the arithmetic progression to inequalities, see [12], [3], [10], [14] and [29]. Generalizations of some of these systems in the sense of Theorem 5 appear in [6], [13], [10], and [27]. This list is far from being a complete list of references. However, we do not have general theorems which give sufficient conditions when a system of equations or inequalities admits a generalization in the sense of a given zero sum theorem. We Conclude with the notion of multiplicity.

Definition 19 (Multiplicity Ramsey numbers for graphs) Let $\mathcal{G}$ be a family of graphs each having $n$ edges and let $r$ and $t$ be positive integers. Denote by $M(\mathcal{G}, r, t)$ 
$\left(M_{E G Z}\left(\mathcal{G}, r, \operatorname{Union}\left(\mathbb{Z}_{n}^{t / 2}\right)\right)\right.$, if $t$ is even and $M_{E G Z}\left(\mathcal{G}, r, U n i o n^{+1}\left(\mathbb{Z}_{n}^{(t-1) / 2}\right)\right)$ if $t$ is odd) the minimum number of graphs that are isomorphic to an element of $\mathcal{G}$, and are monochromatic (the colors of its edges add up to zero), where the minimum is taken over all possible colorings of the edges of $K_{s}$ by $t$ colors. (of the edges of $K_{s}$ by Union $\left(\mathbb{Z}_{n}^{t / 2}\right)$ if $t$ is even and by Union $^{+1}\left(\mathbb{Z}_{n}^{(t-1) / 2}\right)$ if $t$ is odd).

Definition 20 (Multiplicity Rado numbers) Let $x_{1}, x_{2}, \ldots, x_{n k}$ be $n k$ variables partitioned into $k$ sets $S_{i}$, where $i=1,2, \ldots, k$, and suppose that $\left|S_{i}\right|=n$. Furthermore, let $\mathcal{L}$ be a system that consists of equations or inequalities in the variables $x_{1}, x_{2}, \ldots, x_{n k}$. For positive integers $t$ and $r$ denote by $M(\mathcal{L}, r, t) \quad\left(M_{E G Z}\left(\mathcal{L}, r, \operatorname{Union}\left(\mathbb{Z}_{n}^{t / 2}\right)\right)\right.$, if $t$ is even and $M_{E G Z}\left(\mathcal{L}, r\right.$, Union $\left.^{+1}\left(\mathbb{Z}_{n}^{(t-1) / 2}\right)\right)$ if $t$ is odd) the minimum number of $n k$-tuples $x_{1}, \ldots, x_{n k}$ which satisfy $\mathcal{L}$ and each $S_{i}$ is monochromatic, but not necessarily the same color (the colors of the variables in each $S_{i}$ add up to zero), where the minimum is taken over all possible colorings of $1,2, \ldots, r$ by $t$ colors. (of $1,2, \ldots, r$ by Union $\left(\mathbb{Z}_{n}^{t / 2}\right)$ if $t$ is even and by Union $^{+1}\left(\mathbb{Z}_{n}^{(t-1) / 2}\right)$ if $t$ is odd.)

Definition 21 We say that a theorem concerning multiplicity Ramsey numbers, (multiplicity Rado numbers) admits a generalization in the sense of Conjecture 6, if

$M(\mathcal{G}, r, t)=M_{E G Z}\left(\mathcal{G}, r, \operatorname{Union}\left(\mathbb{Z}_{n}^{t / 2}\right)\right)$, if $t$ is even

and $M(\mathcal{G}, r, t)=M_{E G Z}\left(\mathcal{G}, r\right.$, Union $\left.^{+1}\left(\mathbb{Z}_{n}^{(t-1) / 2}\right)\right)$ if $t$ is odd,

$\left(M(\mathcal{L}, r, t)=M_{E G Z}\left(\mathcal{L}, r, \operatorname{Union}\left(\mathbb{Z}_{n}^{t / 2}\right)\right)\right.$, if $t$ is even

and $M(\mathcal{L}, r, t)=M_{E G Z}\left(\mathcal{L}, r\right.$, Union $\left.^{+1}\left(\mathbb{Z}_{n}^{(t-1) / 2}\right)\right)$ if $t$ is odd. $)$

\section{Acknowledgement}

The author would like to thank Bradly Walker for his devotion in programming and obtaining all the experimental results quoted in this paper.

\section{References}

[1] S.D.Adhikari, Y.G. Chen,J.B. Friedlander, S.V. Konyagin, F. Pappalardi, Contributions to zero-sum problems, Discrete Math. 306 (2006), no. 1, 1-10.

[2] N. Alon and M. Dubiner, Zero-sum sets of prescribed size, Combinatorics, Paul Erdős is eighty, Vol. 1, Bolyai Soc. Math. Stud., Janos Bolyai Math. Soc., (Budapest, 1993), 33-50.

[3] N. Alon and J. Spencer, Ascending waves. J. Combin. Theory Ser. A 52 (1989), no. 2, 275-287.

[4] P. Baginski, A generalization of a Ramsey-type theorem on hypermatchings, J. Graph Theory 50 (2005), no. 2, 142-149. 
[5] A. Bialostocki, Some combinatorial number theory aspects of Ramsey theory, research proposal, 1989.

[6] A. Bialostocki, G. Bialostocki, and D. Schaal, A zero-sum theorem, J. Combin. Theory Ser. A, 101 (2003), no. 1, 147-152.

[7] A. Bialostocki and P. Dierker, On the Erdős-Ginzburg-Ziv theorem and the Ramsey numbers for stars and matchings. Discrete Math. 110 (1992), no. 1-3, 1-8.

[8] A. Bialostocki, P. Dierker, On zero sum Ramsey numbers: multiple copies of a graph. J. Graph Theory, 18 (1994), no. 2, 143-151.

[9] A. Bialostocki, P. Dierker, D. Grynkiewicz and M. Lotspeich, On some developments of the ErdősGinzburg-Ziv theorem. II. Acta Arith. 110 (2003), no. 2, 173-184.

[10] A. Bialostocki, P. Erdős, and H. Lefmann, Monochromatic and zero-sum sets of nondecreasing diameter, Discrete Math., 137 (1995), no. 1-3, 19-34.

[11] A. Bialostocki and D. Grynkiewicz, On the intersection of two m-sets and the Erdős-Ginzburg-Ziv theorem, to appear.

[12] A. Bialostocki and D. Schaal, On a variation of Schur numbers. Graphs Combin. 16 (2000), no. 2, 139-147

[13] A. Bialostocki R. Sabar and D. Schaal, On zero-sum generalization of a variation of Schur's equation, preprint.

[14] B. Bollobás, P. Erdős and G. Jin, Strictly ascending pairs and waves, Graph theory, combinatorics, and algorithms, Vol. 1, 2 (Kalamazoo, MI, 1992), 83-95, Wiley-Intersci. Publ., Wiley, New York, 1995.

[15] B. Bollobás and I. Leader, The number of $k$-sums modulo $k$. J. Number Theory 78 (1999), no. 1, 27-35.

[16] Y. Caro, Zero-sum subsequences in abelian non-cyclic groups, Israel J. Math. 92 (1995), no. 1-3, 221233.

[17] Y. Caro, Zero-sum problems-a survey, Discrete Math., 152 (1996), no. 1-3, 93-113.

[18] Y. Edel, C. Elsholtz, A. Geroldinger, S. Kubertin, L. Rackham, Zero-sum problems in finite abelian groups and affine caps, preprint.

[19] C. Elsholtz, Lower bounds for multidimensional zero sums, Combinatorica 24 (2004), no. 3, 351-358.

[20] P. Erdős, A. Ginzburg, and A. Ziv, Theorem in additive number theory, Bull. Res. Council Israel 10F (1961), 41-43.

[21] P. Erdős and R. L. Graham, Old and new problems and results in combinatorial number theory, Monographies de L'Enseignement Mathématique 28. Université de Genève, L'Enseignement Mathématique, Genève, 1980. 128 pp.

[22] Z. Füredi, and D. Kleitman, On zero-trees, J. Graph Theory, 16 (1992), 107-120.

[23] Z. Füredi, and D. Kleitman, The minimal number of zero sums, Combinatorics, Paul Erdős is eighty, Vol. 1, 159-172, Bolyai Soc. Math. Stud., János Bolyai Math. Soc., Budapest, 1993.

[24] W. D. Gao, A combinatorial problem on finite abelian groups. J. Number Theory 58 (1996), no. 1, 100-103.

[25] L. Gallardo, G. Grekos, L. Habsieger, F. Hennecart, B. Landreau, and A. Plagne, Restricted addition in $\mathbb{Z} / n \mathbb{Z}$ and an application to the Erdős-Ginzburg-Ziv problem. J. London Math. Soc. (2) 65 (2002), no. $3,513-523$.

[26] R. L. Graham, B. Rothschild and J. H. Spencer, Ramsey theory. Second edition. Wiley-Interscience Series in Discrete Mathematics and Optimization. A Wiley-Interscience Publication. John Wiley \& Sons, Inc., New York, 1990. xii+196 pp. 
[27] D. J. Grynkiewicz, On four colored sets with nondecreasing diameter and the Erdös-Ginzburg-Ziv theorem. J. Combin. Theory Ser. A 100 (2002), no. 1, 44-60.

[28] D. J. Grynkiewicz, An extension of the Erdős-Ginzburg-Ziv theorem to hypergraphs. European J. Combin. 26 (2005), no. 8, 1154-1176.

[29] D. J. Grynkiewicz, On four color monochromatic sets with nondecreasing diameter. Discrete Math. 290 (2005), no. 2-3, 165-171.

[30] D. J. Grynkiewicz, On the number of $m$-term zero-sum subsequences. Acta Arith. 121 (2006), no. 3, 275-298.

[31] D. J. Grynkiewicz, A weighted Erdős Ginzburg Ziv theorem, preprint.

[32] Y. O. Hamidoune, O. Ordaz and A. Ortuño, On a combinatorial theorem of Erdős, Ginzburg and Ziv. Combin. Probab. Comput. 7 (1998), no. 4, 403-412.

[33] F. Hennecart, La fonction de Brakemeier dans le probléme d'Erdős-Ginzburg-Ziv, Acta Arith. 117 (2005), no. 1, 35-50.

[34] N. Hindman and I. Leader, Partition regular inequalities, European J. Combin. 19 (1998), no. 5, 573-578.

[35] Hui-qin Liu and Zhi-guang Wang, A new proof of Erdős-Ginzburg-Ziv theorem, J. Math. Res. Exposition 24 (2004), no. 1, 89-90.

[36] A. Kemnitz On a lattice point problem, Ars Combin. 16b (1983) 151-160.

[37] M. Kisin, The number of zero sums modulo $m$ in a sequence of length $n$, Mathematica 41 (1994), no. 1, 149-163.

[38] B. M. Landman and A. Robertson, Ramsey theory on the integers. Student Mathematical Library, 24. American Mathematical Society, Providence, RI, 2004. xvi+317 pp.

[39] M. B. Nathanson, Additive Number Theory. Inverse Problems and the Geometry of Sumsets, Graduate Texts in Mathematics, 165, Springer-Verlag, (New York, 1996).

[40] O. Ordaz, Oscar and D. Quiroz, The Erdős-Ginzburg-Ziv theorem in abelian non-cyclic groups. Divulg. Mat. 8 (2000), no. 2, 113-119.

[41] C. Reiher, On Kemnitz conjecture concerning lattice-points in the plane, preprint.

[42] M. Schäffler, Partition regular systems of linear inequalities. Doc. Math. 3 (1998), 149-187 (electronic).

[43] A. Schrijver and P. D. Seymour, A simpler proof and a generalization of the zero-trees theorem, $J$. Combin. Theory Ser. A 58 (1991), no. 2, 301-305.

[44] Zhi-Wei Sun, Unification of zero-sum problems, subset sums and covers of $\mathbb{Z}$. Electron. Res. Announc. Amer. Math. Soc. 9 (2003), 51-60 (electronic).

[45] S. Svetoslav and C. Fang, Kemnitz' conjecture revisited, Discrete Math 297 (2005), no. 1-3, 196-201.

[46] R. Thangadurai, Non-canonical extensions of Erdős-Ginzburg-Ziv theorem. Integers 2 (2002), Paper A7, 14 pp. (electronic).

[47] C. Wang, Note on a variant of the Erdős-Ginzburg-Ziv problem, Acta Arith. 108 (2003), no. 1, 53-59.

[48] J. Zhuang and W. Gao, Erdős-Ginzburg-Ziv theorem for dihedral groups of large prime index. European J. Combin. 26 (2005), no. 7, 1053-1059. 